\title{
ANALYSIS OF MOTION OF SUBSTANCE IN CHANNEL OF NETWORK IN PRESENCE OF PUMPING
}

\author{
NikOLAY K. VitANOV ${ }^{1 *}$, RUMEN BORISOV ${ }^{1}$, \\ TSVETELINA IVANOVA ${ }^{1}$, ZLATINKA I. Dimitrova ${ }^{2}$ \\ ${ }^{1}$ Institute of Mechanics, Bulgarian Academy of Sciences, \\ Acad. G. Bonchev. Str., Bl. 4, 1113 Sofia, Bulgaria \\ ${ }^{2}$ G. Nadjakov Institute of Solid State Physics, Bulgarian Academy of Sciences, \\ Blvd. Tzarigradsko Chaussee 72, 1784 Sofia, Bulgaria \\ [Received: 02 April 2020. Accepted: 05 October 2020] \\ doi: 10.7546/JTAM.50.20.04.03
}

\begin{abstract}
We study motion of substance in a channel of network in presence of possibility for inflow of substance to nodes of the channel. Stationary state of motion of substance can exist in this case and we discuss conditions for existence of such state. We obtain probability distribution connected to distribution of substance in nodes of channel.
\end{abstract}

KEY WORDS: flows in network, network, probability distributions, Waring distribution

\section{INTRODUCTION}

Complex systems attract continuously attention of researchers [1-4]. Just one recent example is connected to influence of COVID-19 on different aspects of human societies. Many complex systems are connected to fluid mechanics or to flows of various substances [5-8]. Especially interesting are flows of substances in systems which structure can be modeled by networks [9-12]. In many cases the flows of substances in such systems can be modeled by systems of differential equations [13,14]. Just one example for such system is connected to models of human migration [15-19]. Migrant flows may be modelled by deterministic or stochastic tools [20,21] and human migration is closely connected to ideological struggles $[22,23]$ and waves and statistical distributions in population systems [24-27].

Below we consider a model of a flow of substance in a channel a network. The channel contains network's nodes and these nodes are considered as boxes (cells) where the following processes can happen: inflow/outflow of substance from/to other nodes of the channel, and "pumping" (inflow of substance from network or environment of network to corresponding node of channel). The different nodes of the channel are assumed to have different rate of "pumping".

The paper is organized as follows. In Section 2 the model for motion of substance in a channel is discussed. Two regimes of functioning of the channel: stationary

\footnotetext{
${ }^{*}$ Corresponding author e-mail: vitanov@imbm.bas.bg
} 
regime and non-stationary regime are described. Probability distribution connected to the amount of substance in the nodes of the channel is obtained. A particular case of distribution for the stationary regime of functioning of the channel is the Waring distribution. In Section 3 we discuss a possible application of the discussed model. Several concluding remarks are summarized in Section 4.

\section{Mathematical Model of the Problem}

Inspired by the models in [31-33] we consider a model of motion of a substance tn a channel as follows. The studied channel contains single arm and this arm has infinite number of nodes. Each node can be considered as a cell (box) and the cells are indexed in succession by non-negative integers. The first cell has index 0 . We assume that an amount $x$ of some substance is distributed among the cells and this substance can move between the cells. Let $x_{i}$ be the amount of the substance in the $i$-th cell. Then

$$
x=\sum_{i=0}^{\infty} x_{i}
$$

The fractions $y_{i}=x_{i} / x$ are considered as probability values of distribution of a discrete random variable $\zeta$

$$
y_{i}=p(\zeta=i), i=0,1, \ldots
$$

The content $x_{i}$ of any cell can change because of 3 processes:

1. Some amount $s$ of the substance $x$ enters the channel from the external environment through the 0 -th cell;

2. Amount $f_{i}$ from $x_{i}$ is transferred from the $i$-th cell into the $i+1$-th cell;

3. Amount $g_{i}$ enters the $i$-th cell from the external environment. This is the difference to previous research where we studied the leakage of substance (the substance leaves the cell and moves to environment. Here we have the opposite process which we call pumping).

These processes can be modeled mathematically by the system of ordinary differential equations:

$$
\begin{aligned}
& \frac{d x_{0}}{d t}=s-f_{0}-g_{0} \\
& \frac{d x_{i}}{d t}=f_{i-1}-f_{i}-g_{i}, \quad i=1,2, \ldots
\end{aligned}
$$


The following forms of the amount of the moving substances are assumed ( $\alpha, \beta, \gamma_{i}$, $\sigma$ are constants)

$$
s=\sigma x_{0} ; \quad \sigma>0
$$

(4) $f_{i}=(\alpha+\beta i) x_{i} ; \quad \alpha>0, \beta \geq 0 \rightarrow$ advantage of cells with large numbers;

$g_{i}=\gamma_{i} x_{i} ; \quad \gamma_{i} \leq 0 \rightarrow$ non-uniform pumpimg over the cells.

We note that the advantage of the cells with larger numbers can be removed by setting $\beta=0$. In addition we note that (i) $s$ is proportional to the the amount of the substance $x_{0}$ in the 0-th node. In [31] $s$ is proportional to the amount $x$ of the substance in the entire channel; (ii) Pumping rates $\gamma_{i}$ are different for the different nodes. In [31] and [33] instead of pumping we have a leakage and the leakage rate is constant and equal to $\gamma$ for all nodes of the channel (i.e., there is uniform leakage over the cells).

Substitution of Eqs. (4) in Eqs. (3) leads to the relationships

$$
\begin{aligned}
& \frac{d x_{0}}{d t}=\sigma x_{0}-\alpha x_{0}-\gamma_{0} x_{0} \\
& \frac{d x_{i}}{d t}=[\alpha+\beta(i-1)] x_{i-1}-\left(\alpha+\beta i+\gamma_{i}\right) x_{i} ; \quad i=1,2, \ldots
\end{aligned}
$$

There are two regimes of functioning of the channel: stationary regime and nonstationary regime.

\subsection{STATIONARY REGIME OF FUNCTIONING OF THE CHANNEL}

In the stationary regime of the functioning of the channel $\sigma=\alpha+\gamma_{0}$ which means that $x_{0}$ (the amount of the substance in the 0-th cell of the channel) is free parameter. We note that all $\gamma_{i}$ are negative and as $\sigma$ can not be negative then $\left|\gamma_{0}\right| \leq \alpha$. If this condition is not fulfilled then the stationary regime of functioning of channel can not be realized.

Let us assume that above condition is fullfilled. In this case the solution of Eqs. (5) is

$$
x_{i}=x_{i}^{*}+\sum_{j=0}^{i} b_{i j} \exp \left[-\left(\alpha+\beta j+\gamma_{j}\right) t\right],
$$

where $x_{i}^{*}$ is the stationary part of the solution. We hote that $\alpha+\beta j+\gamma_{j}$ must be larger than 0 if we want to have a decrease of exponential function in (6) with increasing time. This means that $\alpha+\beta j>-\gamma_{j}$ which imposes another condition on the proces of pumping. The inflow of sbstance to any of the nodes of the channel has an upper limit and if the inflows of substances in all nodes are below these limits then the stationary state of motion of substance is possible. 
For $x_{i}^{*}$ one obtains the relationship

$$
x_{i}^{*}=\frac{\alpha+\beta(i-1)}{\alpha+\beta i+\gamma_{i}} x_{i-1}^{*} .
$$

The corresponding relationships for the coefficients $b_{i j}$ are

$$
b_{i j}=\frac{\alpha+\beta(i-1)}{\gamma_{i}-\gamma_{j}+\beta(i-j)} b_{i-1, j}, \quad j=0,1, \ldots, i-1 .
$$

Here $\gamma_{i}-\gamma_{j}+\beta(i-j) \neq 0$. From Eq. (7) one obtains

$$
x_{i}^{*}=\frac{[k+(i-1)] !}{(k-1) ! \prod_{j=1}^{i}\left(k+j+a_{j}\right)} x_{0}^{*},
$$

where $k=\alpha / \beta$ and $a_{j}=\gamma_{j} / \beta$. The form of the corresponding stationary distribution $y_{i}^{*}=x_{i}^{*} / x^{*}$ (where $x^{*}$ is the amount of the substance in all of the cells of the channel) is

$$
y_{i}^{*}=\frac{[k+(i-1)] !}{(k-1) ! \prod_{j=1}^{i}\left(k+j+a_{j}\right)} y_{0}^{*} .
$$

Let us consider the particular case where $a_{0}=a_{1}=\cdots=a$. In this case the distribution from Eq. (10) is reduced to the distribution:

$$
P(\zeta=i)=P(\zeta=0) \frac{(k-1)^{[i]}}{(a+k)^{[i]}} ; \quad k^{[i]}=\frac{(k+i) !}{k !} ; \quad i=1,2, \ldots
$$

$P(\zeta=0)=y_{0}^{*}=x_{0}^{*} / x^{*}$ is the percentage of substance that is located in the first cell of the channel.

The obtained distribution has interesting particular case Let the percentage of substance located in the entry cell of the channel be

$$
y_{0}^{*}=\frac{a}{a+k} .
$$

The case described by Eq. (16) corresponds to the situation where the amount of substance in the first cell is proportional of the amount of substance in the entire channel (self-reproduction property of the substance). In this case Eq. (10) is reduced to the distribution:

$$
P(\zeta=i)=\frac{a}{a+k} \frac{(k-1)^{[i]}}{(a+k)^{[i]}} ; \quad k^{[i]}=\frac{(k+i) !}{k !} ; \quad i=1,2, \ldots
$$

Distribution (13) is the Waring distribution [28], 


\section{Discussion}

The model discussed in this article can be used to study flow of substances in systems with network structure and in presence of inflow of substance at selected places of corresponding system. Examples for such systems can be water channels or sewer systems. From this point of view parameters of the discussed model can be interpreted as follows. $\sigma$ can be considered as a "gate" parameter as it regulates the amount of substance which enters the channel. Large value of $\sigma$ may lead to large flow of substance through the channel. Second "gate" parameter is the parameter $\alpha$ which regulates the amount of substance which from one part to the next part of the system. Small value of $\alpha$ means that transfer between neighbor parts of the system is more difficult.

Parameter $\beta$ accounts for the tendency that cells numbered by larger numbers can contain more substance. Large value of this parameter means that larger amounts of substance can be concentrated in nodes which are far from the origin of the channel. Parameters $\gamma_{i}$ are the most interesting parameter in our study. In previous studies the value of these parameter were positive, in other words, we considered situation in which substance flows out of cells of the channel (leakage). In this study the value of parameter is negative which means that there is an inflow of substance to the cells of the channel. The changed sign of the parameters $\gamma_{i}$ leads to additional efforts for analysis of situation: because of inflow of substance the stationary regime of flow of substance in the channel may become impossible. The accounting of this leads to several inequalities which have to be fulfilled in order to ensure the existence of the stationary regime of flow.

The negative value of parameters $\gamma_{i}$ leads to interesting possibilities for the shape of probability distribution connected to distribution of substance in the channel. From Eq. (10) we obtain

$$
\frac{y_{i}^{*}}{y_{i+1}^{*}}=1+\frac{\beta+\gamma_{i+1}}{\alpha+\beta i} .
$$

If $\gamma_{i+1}$ was positive (14) leads us to the conclusion that the amount of substance in $i$-th node of the channel has to decrease with increasing $i$. But for the case discussed above $\gamma_{i+1}$ is negative. In addition we have $\alpha+\beta i>-\gamma_{i}$ as condition for existence of stationary flow in the channel. We can write (10) also as

$$
\frac{y_{i+1}^{*}}{y_{i}^{*}}=\frac{\alpha+\beta i}{\alpha+\beta i+\left(\beta+\gamma_{i+1}\right)} .
$$

The sign of $\beta+\gamma_{i+1}$ is important. If $\left|\gamma_{i+1}\right|<\beta$ then the value of $y_{i}$ decreases with increasing $i$. Otherwise the value of $y_{i}$ will increase with increasing $i$ and stationary state is impossible. 
We can write (15) also in the following form:

$$
\frac{y_{i+1}^{*}}{y_{i}^{*}}=\frac{\alpha+\beta i}{\left.\alpha+\beta i+\left(\beta+\mid \gamma_{i+1}\right) \mid+\theta_{i+1}\right)},
$$

where $\theta_{i+1}=0$ for the case when the sign of $\gamma_{i}$ is positive (case of leakage of substance) and $\theta_{i+1}=-2\left|\gamma_{i+1}\right|$ for the case when the sign of $\gamma_{i}$ is negative (case of pumping of substance). (16) shows that in general for the case of pumping the ratio and when stationary state of motion of substance in the channel is possible $\frac{y_{i+1}^{*}}{y_{i}^{*}}$ decreases slowly for the case of pumping in comparison to the case of leakage. Thus one can expect that the parts of the network system which are far from the first segments of the system will be more loaded by substance for the case of pumping in comparison to the case of leakage and this could lead to increased maintenance costs.

\section{CONCLUding REMARKS}

In this article we consider a flow of substance in a channel of network and our attention was concentrated on the case of a channel having single arm and on the case when the substance can flow into the nodes of the channel from the environment. The inflow (called pumping) can lead in many cases to oscillation or permanent increasing of the amount of substance in the channel but there are conditions where the flow of substance in the channel can be stationary. Such kind of flow is studied above and probability distribution connected to the corresponding distribution of substance in the nodes of the channel is obtained. The obtained distribution contains as particular case the long-tail Waring distributions and the comparison to the case of outflow of substance from nodes of channel (leakage) shows that for the same values of parameters $\alpha$ and $\beta$ there can be more substance in nodes far from channel entrance for case of pumping in comparison to case of leakage. In other words the long tail of the distribution can become also more "fat" one.

\section{ACKNOWLEDGEMENTS}

This research was partially supported by the project BG05M2OP001-1.001-0008 "National Center for Mechatronics and Clean Technologies", funded by the Operating Program "Science and Education for Intelligent Growth" of Republic of Bulgaria and by the National Scientific Program "Information and Communication Technologies for a Single Digital Market in Science, Education and Security" (ICTinSES), contract No D01205/23.11.2018, financed by the Ministry of Education and Science in Bulgaria. 


\section{REFERENCES}

[1] N. BoccarA. Modeling Complex Systems (2004) Springer, New York.

[2] P. Fieguth (2017) An Introduction to Complex Systems: Society, Ecology, and Nonlinear Dynamics. Springer International Publishing, Switzerland.

[3] H. Kantz, D. Holstein, M. Ragwitz, N.K. Vitanov (2004) Markov Chain Model for Turbulent Wind Speed Data. Physica A 342 315-321.

[4] K.T. Ashenfelter, S.M. Boker, J.R. Waddell, N. Vitanov (2009). Spatiotemporal Symmetry and Multifractal Structure of Head Movements During Dyadic Conversation. Journal of Experimental Psychology: Human Perception and Performance 35 1072-1091.

[5] P. MannevilLe (2004) Instabilities, Chaos, and Turbulence: An Introduction to Nonlinear Dynamics and Complex Systems. Imperial College Press, London.

[6] S.E. Spagnolie (2015) Complex Fluids in Biological Systems: Experiment, Theory, and Computation. Springer, New York.

[7] T. Boeck, N.K. Vitanov (2002). Low-Dimensional Chaos in Zero-Prandtl-Number BenardMarangoni Convection. Physical Review E, 65037203.

[8] N.K. Vitanov, A. Chabchoub, N. Hoffmann (2013). Deep-Water Waves: On the Nonlinear Schrödinger Equation and its Solutions. Journal of Theoretical and Applied Mechanics, 43, No.2 43-54.

[9] G. Chen, X. Wang, X. Li (2015) Fundamentals of Complex Networks: Models, Structures and Dynamics. Wiley, Singapore.

[10] D. Stephenson (1998) Water Supply Management. Springer, Dordrecht.

[11] N.K. Vitanov, K.N. Vitanov (2019). Statistical Distributions Connected to Motion of Substance in a Channel of a Network. Physica A 527, 121174.

[12] N.K. Vitanov, K.N. Vitanov (2018). Discrete-Time Model for a Motion of Substance in a Channel of a Network with Application to Channels of Human Migration. Physica A, 509 635-650.

[13] A. Bressan, S. Canic, M. Garavello, M. Herty, B. Piccoli (2014) Flows on Networks: Recent Results and Perspectives. EMS Surveys in Mathematical Sciences, 1 $47-111$.

[14] N.K. Vitanov, K.N. Vitanov (2018). On the Motion of Substance in a Channel of a Network and Human Migration. Physica A 490 1277-1294.

[15] E.S. LEE (1966) A Theory of Migration. Demography 3 47-57.

[16] J.T. FAWCET (1989) Networks, Linkages, and Migration Systems. International Migration Review 23 671-680.

[17] W.J. EthieR (1985) International Trade and Labor Migration. The American Economic Review 75 691-707.

[18] J.R. HARRIS, M.P. Todaro (1970) Migration, Unemployment and Development: A Two-Sector Analysis. The American Economic Review 60 126-142.

[19] R. Skeldon (1992) Migration and Development: A Global Perspective. Routledge, London. 
[20] Hotelling H (1978) A Mathematical Theory of Migration. Environment and Planning 10 1223-1239.

[21] T. PUU (1991) Hotelling's Migration Model Revisited. Environment and Planning 23 1209-1216.

[22] N.K. Vitanov, Z.I. Dimitrova, M. Ausloos (2010) Verhulst-Lotka-Volterra model of ideological struggle. Physica A 389 4970-4980.

[23] N.K. Vitanov, M. Ausloos, G. Rotundo (2012) Discrete Model of Ideological Struggle Accounting for Migration. Advances in Complex Systems 15, Supplement 1, Article number 1250049.

[24] N.K. Vitanov, K. N. Vitanov Population Dynamics in Presence of State Dependent Fluctuations (2013) Computers \& Mathematics with Applications 68 962-971.

[25] N.K. Vitanov, I.P. Jordanov, Z.I. Dimitrova (2009) On Nonlinear Dynamics of Interacting Populations: Coupled Kink Waves in a System of Two Populations. Communications in Nonlinear Science and Numerical Simulation 14 2379-2388.

[26] N.K. Vitanov, I.P. Jordanov, Z.I. Dimitrova (2009) On Nonlinear Population Waves. Applied Mathematics and Computation 215 2950-2964.

[27] N.K. Vitanov, Z.I. Dimitrova, K.N. Vitanov (2013) Traveling Waves and Statistical Distributions Connected to Systems of Interacting Populations. Computers \& Mathematics with Applications 66 1666-1684.

[28] V. Diodato (1994) Dictionary of Bibliometrics. Haworth Press, Binghampton, NY (1994).

[29] J.O. IRWIN (1963) The Place of Mathematics in Medical and Biological Sciences. Journal of the Royal Statistical Society 126 1-44.

[30] J.O. IRwIN (1968) The Generalized Waring Distribution Applied to Accident Theory. Journal of the Royal Statistical Society 131 205-225.

[31] A. Schubert, W. GlänZel (1984) A Dynamic Look at a Class of Skew Distributions. A Model with Scientometric Application. Scientometrics 6 149-167.

[32] N. K. Vitanov (2016) Science Dynamics and Research Production: Indicators, Indexes, Statistical Laws and Mathematical Models. Springer, Cham.

[33] N.K. Vitanov, K.N. Vitanov (2016) Box Model of Migration Channels. Mathematical Social Sciences 80 108-114. 